\title{
THE NUMBER OF GENERATORS OF MODULES OVER POLYNOMIAL RINGS
}

\author{
GENNADY LYUBEZNIK
}

(Communicated by Louis J. Ratliff, Jr.)

\begin{abstract}
Let $k$ be an infinite field and $B=k\left[X_{1}, \ldots, X_{n}\right]$ a polynomial ring over $k$. Let $M$ be a finitely generated module over $B$. For every prime ideal $P \subset B$ let $\mu\left(M_{P}\right)$ be the minimum number of generators of $M_{P}$, i.e., $\mu\left(M_{P}\right)=$ $\operatorname{dim}_{B_{P} / P_{P}}\left(M_{P} \otimes_{B_{P}}\left(B_{P} / P_{P}\right)\right)$. Set $\eta(M)=\max \left\{\mu\left(M_{P}\right)+\operatorname{dim}(B / P) \mid P \in\right.$ Spec $B$ such that $M_{P}$ is not free\}. Then $M$ can be generated by $\eta(M)$ elements. This improves earlier results of A. Sathaye and N. Mohan Kumar on a conjecture of Eisenbud-Evans.
\end{abstract}

Given a finitely generated module $M$ over a commutative ring $B$, one would like to determine $\mu(M)$, the minimum number of generators of $M$.

This problem goes back to Serre (as mentioned in [Sw2]). $B$.

Of course, if $B$ is local, $\mu(M)=\operatorname{dim}_{k}\left(M \otimes_{B} k\right)$, where $k$ is the residue field of

If $B$ is not local, for every prime ideal $P \subset B$ one sets

$$
b_{P}(M)= \begin{cases}\mu\left(M_{P}\right)+\operatorname{dim}(B / P) & \text { if } M_{P} \neq 0 \\ 0 & \text { if } M_{P}=0 .\end{cases}
$$

The Forster-Swan theorem [F, Sw1], states that $\mu(M)$ is less than or equal to the maximum of $b_{P}(M)$ as $P$ runs through all $j$-primes of $B$. A $j$-prime is a prime which is an intersection of maximal ideals.

If $B=A[X]$, A. Sathaye [Sa] and N. Mohan Kumar [MK] proved that $M$ can be generated by $\max \left\{b_{P}(M) \mid P \in \operatorname{Spec} B\right.$ such that $\left.\operatorname{dim}(B / P)<\operatorname{dim} B\right\}$ elements.

In this short note we considerably improve the Sathaye-Mohan Kumar estimate in the case when $B$ is a polynomial ring in $n$ variables over an infinite field $k$. Namely we show that in this case it is enough to take the maximum of $b_{P}(M)$ only over those prime ideals of $B$ at which $M$ is not free (Theorem 2).

For example, if $M$ is not free at a unique maximal ideal at which it can be generated by $d$ elements, our results show that $M$ can be generated by $d$ elements, while the Sathaye-Mohan Kumar results only show that $M$ can be generated by $\max \{d, r+n-1\}$ elements, where $r$ is the rank of $M$.

N. Mohan Kumar also proved in the same paper that if $B=k\left[X_{1}, \ldots, X_{n}\right]$ and $I \subset B$ an ideal such that $I / I^{2}$ is $d$-generated and $d \geq 2+\operatorname{dim}(B / I)$, then $I$ is $d$-generated.

Received by the editors May 11, 1987 and, in revised form, July 21, 1987.

1980 Mathematics Subject Classification (1985 Revision). Primary 13B25, 13 C99.

National Science Foundation Postdoctoral Fellow. 
This result of Mohan Kumar is a special case (when $M=I$ ) of the following

THEOREM 1. Let $B=k\left[X_{1}, \ldots, X_{n}\right]$, where $k$ is an infinite field and $M a$ finitely generated $B$-module of rank $r$. Let $I$ be the defining ideal of the set of primes of $B$ at which $M$ is not free. Assume $M / I M$ can be generated by $d$ elements. If $d \geq 1+r+\operatorname{dim}(B / I)$, then $M$ can be generated by $d$ elements.

PROOF OF THEOREM 1. Let $f_{1}, \ldots, f_{s}$ be some generators of $I$. For every finitely generated $B$-module $N$ set

$$
b\left(N_{f_{i}}\right)=\max \left\{b_{P}\left(N_{f_{i}}\right) \mid P \text { a prime in } B_{f_{i}}\right\}
$$

and set

$$
b^{I}(N)=\max \left\{b\left(N_{f_{i}}\right) \mid i=1, \ldots, s\right\} .
$$

Clearly

$$
b^{I}(N)=\max \left\{b_{P}(N) \mid P \in \operatorname{Spec} B, P \not \supset I\right\} .
$$

Thus, $b^{I}(M)=r+n$.

Let $a_{1}, \ldots, a_{d}$ be elements of $M$ which generate $M / I M$. We claim there exist $a_{1}^{\prime}, \ldots, a_{d}^{\prime} \in M$ such that they also generate $M / I M$ and $b^{I}\left(M /\left(B a_{1}^{\prime}+\cdots+B a_{d}^{\prime}\right)\right) \leq$ $b^{I}(M)-d=r+n-d$.

For every $t$ set $N_{t}=M /\left(B a_{1}^{\prime}+\cdots+B a_{t}^{\prime}\right)$.

The elements $a_{1}^{\prime}, \ldots, a_{d}^{\prime}$ will be constructed by induction on $t$ subject to the condition that $N_{t} / I N_{t}$ be generated by $(d-t)$ elements and $b^{I}\left(N_{t}\right) \leq b^{I}(M)-t$ for every $t$.

Assume $a_{1}^{\prime}, \ldots, a_{t}^{\prime}$ have been found and let $a_{t+1} \in M$ form part of a generating set of $N_{t} / I N_{t}$ consisting of $d-t$ elements. Then any element $a_{t+1}^{\prime}=a_{t+1}+\lambda y$, where $\lambda \in I$, and $y \in M$ also will form part of a system of generators of $N_{t} / I N_{t}$ consisting of $d-t$ elements. Since $N_{t+1} / I N_{t+1}=N_{t} /\left(B a_{t+1}^{\prime}+I N_{t}\right)=\left(N_{t} / I N_{t}\right) / B a_{t+1}^{\prime}$, the module $N_{t+1} / I N_{t+1}$ will be generated by $d-t-1$ elements.

To ensure that $b^{I}\left(N_{t+1}\right) \leq b^{I}\left(N_{t}\right)-1$ it is enough to ensure that $b\left(\left(N_{t+1}\right)_{f_{i}}\right) \leq$ $b\left(\left(N_{t}\right)_{f_{i}}\right)-1$ for every $i$. It follows from the proof of the main theorem of [F] that in order that $b\left(\left(N_{t+1}\right)_{f_{i}}\right) \leq b\left(\left(N_{t}\right)_{f_{i}}\right)-1$ it is necessary and sufficient that the element $a_{t+1}^{\prime}$ be basic for $\left(N_{t}\right)_{f_{i}}$ at some finitely many primes of $B_{f_{i}}$ (namely, at those primes where $b_{P}\left(\left(N_{t}\right)_{f_{i}}\right)=b\left(\left(N_{t}\right)_{f_{i}}\right)$; there are only finitely many of those). Summarizing, we see that in order that $b^{I}\left(N_{t+1}\right) \leq b^{I}\left(N_{t}\right)-1$ it is sufficient to make $a_{t+1}^{\prime}$ basic for $N_{t}$ at some finite set of primes $P_{1}, \ldots, P_{v}$ of $B$ not one of which contains $I$.

Let $y \in M$ be an element basic for $N_{t}$ at $P_{1}, \ldots, P_{v}$. It exists by Hilfssatz [F]. Since $P_{i} \not \supset I$ for every $i$, we can find $\lambda \in I \backslash\left(P_{1} \cup \cdots \cup P_{v}\right)$ such that $a_{t+1}^{\prime}=a_{t+1}+\lambda y$ is basic at $P_{1}, \ldots, P_{v}$. This proves the claim.

Set $N=N_{d}=M /\left(B a_{1}^{\prime}+\cdots+B a_{d}^{\prime}\right)$ and set $J=\operatorname{ann}(N)$. We claim that $\operatorname{dim}(B / I)+\operatorname{dim}(B / J) \leq n-2$. Indeed, let $P$ be a minimal prime over-ideal of $J$. Then $\mu\left(N_{P}\right) \geq 1$ and $b_{P}(N)=\mu\left(N_{P}\right)+\operatorname{dim}(B / P) \leq r+n-d$, which implies $\operatorname{dim}(B / P) \leq r+n-d-\mu\left(N_{P}\right) \leq r+n-d-1$.

Since $d \geq 1+r+\operatorname{dim}(B / I)$, we see that $\operatorname{dim}(B / I) \leq d-r-1$ and therefore $\operatorname{dim}(B / I)+\operatorname{dim}(B / J) \leq r+n-d-1+d-r-1 \leq n-2$.

Since $I$ belongs to the radical of $B_{1+I}$ and $a_{1}^{\prime}, \ldots, a_{d}^{\prime}$ generate $M / I M$, by Nakayama's lemma they generate $M_{1+I}$. So $N_{1+I}=0$ and therefore $J$ contains an element from $1+I$. This implies $I+J=B$. 
Let $L$ be the kernel of the map $\phi: B^{d} \rightarrow M$ which sends the $i$ th free generator of $B^{d}$ to $a_{i}^{\prime}$. Let $f \in B$ be an element such that $L_{f}$ is free over $B_{f}$. We claim there exist new variables $x_{1}, \ldots, x_{n}$ such that after putting $A=k\left[x_{1}, \ldots, x_{n-1}\right]$ we will have

(1) $(I \cap A)+(J \cap A)=1$;

(2) $f$ is monic in $x_{n}$.

In fact, a generic linear change will do the trick. Indeed, it is well known that any $f$ becomes monic in $x_{n}$ after a generic linear change.

Now we have to show that a generic projection has the property that $(I \cap A)+$ $(J \cap A)=1$. But the algebraic set consisting of all lines joining every point of the algebraic set defined by $I$ to every point of the algebraic set defined by $J$ has dimension at most $\operatorname{dim}(B / I)+\operatorname{dim}(B / J)+1 \leq n-2+1=n-1$, so, the intersection of this set of lines with the hyperplane at infinity has dimension $\leq n-2$. Since the hyperplane at infinity has dimension $n-1$, it contains points which do not belong to this set and they form a Zarisky open subset. A projection from one of those points will do. Since a generic linear change satisfies (1) and (2), there exists a linear change which satisfies both (1) and (2).

So, let $s \in I \cap A$ and $s^{\prime} \in J \cap A$ be such that $s+s^{\prime}=1$. Then $a_{1}^{\prime}, \ldots, a_{d}^{\prime}$ generate $M_{s^{\prime}}$ and the map $\phi_{s^{\prime}}: B_{s^{\prime}}^{d} \rightarrow M_{s^{\prime}}$ is surjective. The kernel of $\phi_{s^{\prime}}$ is $L_{s^{\prime}}$. Since $s \in I$, we see that $M_{s s^{\prime}}$ is projective, so $\phi_{s s^{\prime}}: B_{s s^{\prime}}^{d} \rightarrow M_{s^{\prime} s}$ splits and $L_{s s^{\prime}}$ is projective too. Since $L_{s s^{\prime}}$ becomes free after inverting a monic $f$, it is free of rank $d-r$.

We construct a projective $B$-module $P$ of rank $d$ and a surjection $P \rightarrow M$ as follows. Over $B_{s^{\prime}}$ take $P_{s^{\prime}}$ to be the free module of rank $d$ and surject it into $M_{s^{\prime}}$ by $\phi_{s^{\prime}}: B_{s^{\prime}}^{d} \rightarrow M_{s^{\prime}}$. Over $B_{s}$ take $P_{s}$ to be $M_{s} \oplus B_{s}^{d-r}$ and surject it onto $M_{s}$ by a map $\psi: M_{s} \oplus B_{s}^{d-r} \rightarrow M_{s}$ which is the projection onto the first factor. The surjections $\psi_{s^{\prime}}$ and $\phi_{s^{\prime} s}$ can be patched up over $B_{s^{\prime}}$, since they split and their images are isomorphic to $M_{s s^{\prime}}$ and their kernels are free of rank $d-r$. This patching produces a projective module of rank $d$ which surjects onto $M$. Since all projectives over $B$ are free, $M$ is $d$-generated. Q.E.D.

THEOREM 2. Set $\eta(M)=\max \left\{b_{P}(M) \mid P \in \operatorname{Spec} B\right.$ such that $M_{P}$ is not free $\}$. Then $M$ can be generated by $\eta(M)$ elements.

PROOF. Let $I$ be the defining ideal of the set of primes at which $M$ is not free. Let $P$ be a minimal prime over-ideal of $I$ such that $\operatorname{dim}(B / P)=\operatorname{dim}(B / I)$. Since $M_{P}$ is not free, $\mu\left(M_{P}\right) \geq 1+r$ and therefore $\eta(M) \geq \mu\left(M_{P}\right)+\operatorname{dim}(B / P) \geq$ $1+r+\operatorname{dim}(B / I)$. Since $M / I M$ is a module over $B / I$, it can be generated by $\max \left\{\mu\left((M / I M)_{P}\right)+\operatorname{dim}((B / I) / P) \mid P \in \operatorname{Spec} B / I\right\}$. But this number equals $\eta(M)$. Now we are done by Theorem 1 with $d=\eta(M)$.

REMARKS. A theorem of D. Quillen and A. Suslin says that if $P$ is a projective module over $B=A[T]$ which becomes free after inverting a monic polynomial $f$, then $P$ is free.

Our results suggest the following generalization of the Quillen-Suslin theorem to the nonprojective case.

Conjecture. Let $B=A[T]$ where $A$ is commutative Noetherian and $M$ a finitely generated $B$-module such that $M_{f}$ is $B_{f}$-free of rank $r$ where $f$ is monic in $T$. Let $I$ be the defining ideal of the set of primes of $B$ at which $M$ is not free. If 
$M / I M$ can be generated by $d$ elements and $d \geq 1+r+\operatorname{dim}(B / I)$, then $M$ also can be generated by $d$ elements.

In the special case when $M$ is an ideal of $B$ this has been proven by S. Mandal [Ma].

An interesting problem is to determine, for a given finitely generated module $M$ over some commutative ring $B$, the minimum rank of a $B$-projective which surjects onto $M$.

In particular, one can ask the following

QUESTION. When is $M$ the surjective image of a projective of rank $\eta(M)$ ?

Boratynski [Bo] gives an example of a maximal ideal $M$ in a three-dimensional regular affine algebra over the reals which is not the surjective image of a rank 3 projective (although clearly $\eta(M)=3$ ). So the answer is not always positive.

On the other hand, Murthy [Mu] has shown that the answer is positive for ideals in affine algebras over algebraically closed fields.

Proposition. Let $M$ be a finitely generated $B$-module and $s, s^{\prime} \in B$ elements such that $M_{s}$ is $B_{s}$-projective of rank $r$ and $M_{s^{\prime}}$ is $(r+1)$-generated. Then $M$ is the surjective image of a projective $B$-module of rank $r+1$.

PROOF. Over $B_{s^{\prime}}$ take a free module of rank $(r+1)$ which surjects onto $M_{s^{\prime}}$ and over $B_{s}$ take $M_{s} \oplus\left(\bigwedge^{r} M_{s}\right)^{*}$ with the projection onto the first summand. They patch over $B_{s s^{\prime}}$ and give a projective of rank $r+1$ over $B$ which surjects onto $M$.

With the help of this proposition one can show that the answer to the above question is positive provided $\operatorname{dim} B \leq 2$.

\section{REFERENCES}

[Bo] M. Boratyński, A note on set-theoretic complete intersection ideals, J. Algebra 54 (1978), 1-5.

[F] O. Forster, Über die Anzahl der Erzeugenden eines Ideals in einem Noetherschen Ring, Math. Z. 84 (1964), 80-87.

[Ma] S. Mandal, On efficient generation of ideals, Invent. Math. 75 (1984), 59-67.

[Mu] M. P. Murthy (in preparation).

[MK] N. Mohan Kumar, On two conjectures about polynomials rings, Invent. Math. 46 (1978), 225-236.

[Sw1] R. G. Swan, Vector bundles and projective modules, Trans. Amer. Math. Soc. 105 (1962), 264-277.

[Sw2] _ The number of generators of a module, Math. Z. 102 (1967), 318-322.

[Sa] A. Sathaye, On the Forster-Eisenbud-Evans conjecture, Invent. Math. 46 (1978), 211-224.

Department of Mathematics, University of Chicago, Chicago, Illinois 60637 